\title{
Dual mechanism-based sensing of mercury using unmodified, heteroepitaxially synthesized silver nanoparticles
}

\author{
Amit Nain $^{1} \cdot$ Snigdha Roy Barman ${ }^{1} \cdot$ Saumey Jain ${ }^{1}$ Amitava Mukherjee ${ }^{2}$. \\ Jitendra Satija ${ }^{2}$ (D)
}

Received: 30 April 2017/Accepted: 30 May 2017/Published online: 8 June 2017

(c) The Author(s) 2017. This article is an open access publication

\begin{abstract}
Mercury and its compounds are widely distributed in the environment and have a significant negative impact on human health. In this paper, we report the development of a rapid and facile method for the detection of mercury ions $\left(\mathrm{Hg}^{2+}\right)$ using heteroepitaxially synthesized unmodified silver nanoparticle-based smart probes using UV-Vis spectrophotometer and also through the naked eye by means of a paper-based sensor strip. The silver nanoparticles were prepared by heteroepitaxial growth method using gold seed nanoparticle of $\sim 2.4 \mathrm{~nm}$ size as the template. The silver is grown on the seed particles by reducing the silver-ammonia complex using glucose, which resulted in Glu-AgNPs having an average size of $14.65 \pm 3.53 \mathrm{~nm}$. The sensing of mercury ions was carried out in aqueous solution and the reaction response was monitored by UV-Vis spectrophotometer. The interaction of $\mathrm{Hg}^{2+}$ with Glu-AgNPs resulted in a significant drop in the absorbance at $402 \mathrm{~nm}$ along with a prominent color change (from bright yellow to colorless) and wavelength shift (blue shift). The limit of detection (LOD) of this assay was found to be $100 \mathrm{nM}$ (i.e., $20 \mathrm{ppb}$ ) with a good linearity in the concentration range of $100-10 \mathrm{mM}$. To further ease
\end{abstract}

Amit Nain and Snigdha Roy Barman have contributed equally to this work.

Electronic supplementary material The online version of this article (doi:10.1007/s13204-017-0572-0) contains supplementary material, which is available to authorized users.

Jitendra Satija

jsatija11@gmail.com; jsatija@vit.ac.in

1 School of Biosciences and Technology, VIT University, Vellore, Tamil Nadu 632014, India

2 Centre for Nanobiotechnology, VIT University, Vellore, Tamil Nadu 632014, India the detection process and make it field deployable, we attempted to develop a paper-based sensor strip by immobilizing Glu-AgNPs on a paper strip. Upon interaction with mercury solution of varying concentrations, the decoloration of the spots could be observed easily through naked eyes, with the limit of detection under sub-optical conditions being $1 \mu \mathrm{M}$.

Keywords Optical sensor · Glucose-capped silver nanoparticles · Galvanic etching · Paper based sensor

\section{Introduction}

Accumulation of mercury, in every aspect of environmental samples including water and soil, can cause severe health problems to animal and human beings. Although all the oxidation states of mercury are toxic, $\mathrm{Hg}^{2+}$ form is the most stable and exists primarily in surface water due to its high aqueous solubility. Once introduced into the aquatic ecosystem, it accumulates in the food chain in the form of methylmercury (a neurotoxin), especially in the edible fishes, and can cause prenatal brain damage, various cognitive and motion disorders, and the most deadly Minamata disease (Trasande et al. 2005; Graeme 1998). As per WHO recommendations, the maximum permissible level of mercury in drinking water is $1 \mu \mathrm{g} / \mathrm{L}$. Excess intake of $\mathrm{Hg}^{2+}$ can create serious health risks to human body due to its highly toxic effects on brain, kidney, and immune and nervous systems (Clarkson and Magos 2006; Tchounwou et al. 2003). Therefore, monitoring the $\mathrm{Hg}^{2+}$ ions in aqueous system is highly demanding so as to maintain the environment.

Various traditional analytical methods and instruments are currently available for mercury detection, such as cold 
wave atomic absorption spectroscopy (AAS), gas chromatography-mass spectroscopy (GC-MS), inductively coupled plasma mass spectroscopy (ICP-MS), and highperformance liquid chromatography (HPLC) (Puk and Weber 1994; Vallant et al. 2007; Barin et al. 2014; Han et al. 2006). However, these techniques are highly expensive, and require sophisticated operating facilities, complex sample treatments and skilled personnel, and thus are not suitable for field deployment. Therefore, development of a simple, rapid, highly sensitive, and affordable method/device for selective detection of $\mathrm{Hg}^{2+}$ is still a major pursuit.

Recent advances in the field of nanotechnology have resulted in the development of a wide range of optical and electrochemical sensors for mercury detection (Govindhan et al. 2014; Ding et al. 2016; Alex et al. 2016; Mao et al. 2015; Cui et al. 2015; Sun et al. 2016). These have shown many favorable advantages over the conventional tools, including low cost of analysis, shorter analysis time, field deployability, high specificity, and ultra-sensitivity. Among the different nanomaterials, silver nanoparticles (AgNPs) have garnered more attention for mercury sensor development, mainly due to two reasons: (1) the localized surface plasmon resonance (LSPR) of AgNPs is highly sensitive to micro-level change in refractive index (RI) of their surroundings, causing a prominent shift in their peak absorption wavelength (Martinsson et al. 2014; Guo et al. 2015) and (2) a redox reaction between $\mathrm{Hg}^{2+}$ and $\mathrm{AgNPs}\left(\mathrm{Ag}^{0}\right)$ resulting in $\mathrm{Ag}-\mathrm{Hg}$ amalgam formation by means of etching of nanoparticles, and thus causing a prominent change in the absorbance intensity and spectral peak (Ramesh and Radhakrishnan 2011; Fan et al. 2009; Henglein and Brancewicz 1997).

In the past decade, numerous AgNP-based colorimetric sensing modalities have been developed for mercury detection (Botasini et al. 2013; Ren et al. 2012; Tao et al. 2012). Among these, one common approach is to make use of as-prepared AgNPs along with certain chemicals or biochemicals, either before or during the $\mathrm{Hg}^{2+}$ sensing (Liu et al. 2011; Wang et al. 2010). For example, Duan et al. (2014) designed anti-aggregation-based colorimetric sensor for the detection of $\mathrm{Hg}^{2+}$ ions, which requires the addition of 6-thioguanine during the assay. In the absence of mercury ions, introduction of 6-thioguanine induces the aggregation of AgNPs, resulting in spectral shift and color change from brown to yellow. This can be attributed to the fact that when mercury ions are present, they form a complex with 6-thioguanine, because of their higher affinity towards thiol ligands (Rooney 2007), and thus preventing the aggregation of AgNPs (i.e., anti-aggregation). In a similar fashion, the potential of various other thiol compounds, such as cysteine (Ding et al. 2012) and dithiothreitol (DTT) (Karthiga et al. 2016; Rajeshwari et al. 2016), have also been investigated by various research groups to develop anti-aggregation assays for $\mathrm{Hg}^{2+}$ detection. Although these assays have shown many advantages in terms of sensitivity and selectivity, these methods essentially require some stringent application environment. In other words, most of these analytical methods are very sensitive to change in $\mathrm{pH}$, temperature, and ion concentration and are much suited to lab-based experiments, but these factors might be deterrent to applicability of the AgNP-based nanoanalytical methods in remote locations. In addition, the use of auxiliary chemicals or biochemicals makes these assay procedures complex and increases the overall cost of analysis.

Another important and widely explored approach is to design tailor-made functionalized silver nanoparticles by conjugating them with a specific type of ligand, followed by their complexation with $\mathrm{Hg}^{2+}$ ions. The interaction causes the change in solution color and spectral shift by virtue of either etching of nanoparticles or ligand removal followed by their aggregation. For example, Yuan et al. (2013) utilized dendrimer-stabilized silver nanoparticles (DSAgNPs) to detect $\mathrm{Hg}^{2+}$ in aqueous samples. Authors suggested that in the $\mathrm{pH}$ range of 5-8, primary and tertiary amine group of poly(amidoamine)(PAMAM) dendrimers enabled high local concentration of $\mathrm{Hg}^{2+}$ ions via complexation. This resulted in an efficient redox reaction between $\mathrm{Ag}^{0}$ and $\mathrm{Hg}^{2+}$, transforming the DSAgNPs into smaller size by means of etching. Consequently, a decoloration of the solution and a blue shift in the plasmonic peak along with a significant decrease in the absorbance intensity were observed as a function of $\mathrm{Hg}^{2+}$ concentration. This method again hits a roadblock in the field deployability, as it is most efficient in a particular range of $\mathrm{pH}$, which might require some chemical manipulation before analysis for sensitive and accurate quantitative determination. Few research groups have developed colorimetric sensor by exploiting the affinity reaction between thiol and mercury ions by making use of thiol-capped AgNPs. For example, Bhattacharjee and Chakraborty (2014) demonstrated that $\mathrm{Hg}^{2+}$ ions are thiophilic and bind with thiol groups of cysteamine-capped silver nanoparticles. This causes the removal of cysteamine capping material from the AgNPs surface resulting in uncapped (and thus poorly stabilized) AgNPs, which tend to form aggregates. Simultaneously, a redox reaction between $\mathrm{Ag}^{0}$ and $\mathrm{Hg}^{2+}$ leads to the formation of $\mathrm{Ag}-\mathrm{Hg}$ amalgam resulting in disappearance of AgNPs. Overall, the combined effect modulated the LSPR properties of AgNPs and turned the solution color from yellow to colorless. Although these approaches have many potential advantages, surface functionalization of AgNPs makes the process complex and less user-friendly and may cause batch to batch variation in the sensor performance.

To overcome the aforesaid issues, the use of as-prepared AgNPs for the development of colorimetric assay/scheme, 
without the addition of any external reagents, might be a viable solution for field monitoring of $\mathrm{Hg}^{2+}$. Although few reports are available, these have shown limited sensitivity and selectivity. For instances, Farhadi et al. (2012) reported the use of plant extract-derived AgNPs for mercury sensing. The limit of detection (LOD) of this assay was found to be $2.2 \mu \mathrm{M}$ of $\mathrm{Hg}^{2+}$, while the LOD through naked eye detection, i.e., yellow color to discoloration of colloidal AgNP solution, could be achieved at $100 \mu \mathrm{M}$.

In this study, we demonstrated the direct application of glucose-capped silver nanoparticles (Glu-AgNPs) as a smart probe for label-free detection of $\mathrm{Hg}^{2+}$ in aqueous medium (Scheme 1). The as-prepared Glu-AgNPs, i.e., without any surface modification and without any additional chemical or biochemical reagents, were used for mercury sensing, which is the most important advantage. Another important advantage is the lower limit of detection, i.e., $20 \mathrm{ppb}$ with UV-Vis spectroscopy. Further, we demonstrated the deployability of the assay using a paperbased sensing strip, which could detect $1 \mu \mathrm{M}$ of $\mathrm{Hg}^{2+}$ ions through naked eye under sub-optical conditions.

\section{Materials and methods}

\section{Materials}

Silver nitrate $\left(\mathrm{AgNO}_{3}\right)$ and gold chloride $\left(\mathrm{HAuCl}_{4}\right)$ were purchased from Sigma-Aldrich. Tetrakis(hydroxymethyl)phosphonium chloride (THPC), ammonia solution
$\left(\mathrm{NH}_{3}\right)$, sodium hydroxide $(\mathrm{NaOH})$, and glucose were purchased from Merck Chemicals. All the other reagents and chemicals were of analytical grade and used without further purification. Prior to synthesis of nanoparticles, all the glassware were cleaned with Aqua Regia (3:1 $\left.\mathrm{HCl}: \mathrm{HNO}_{3}\right)$. Milli-Q water was used for cleaning and rinsing of glassware and preparation of all types of solutions.

\section{Synthesis of silver nanoparticles}

Silver nanoparticles were synthesized by heteroepitaxial growth approach as it yields nanoparticles of uniform size and shape (Tharion et al. 2015). The silver was grown on seed gold nanoparticles (AuNPs) by modified Tollens' reaction. First, the seed AuNPs were prepared at room temperature by reducing gold chloride using alkaline solution of THPC as described earlier (Duff et al. 1993). For this, $1 \mathrm{~mL}$ of freshly prepared THPC solution $(0.96 \%$ v/v) was added to $45 \mathrm{~mL}$ of $11 \mathrm{mM}$ aqueous $\mathrm{NaOH}$ solution. After stirring the mixture for $5 \mathrm{~min}, 600 \mu \mathrm{L}$ of aqueous $\mathrm{HAuCl}_{4}$ solution (1\% w/v) was added at once. Instantly, the solution color changed to dark brown indicating the formation of seed AuNPs. After $2 \mathrm{~h}$ of incubation, the seed AuNPs solution was stored at $4{ }^{\circ} \mathrm{C}$ until further use. Transmission electron microscopy (TEM) image analysis revealed the size of the formed seed AuNPs to be $2.39 \pm 0.67 \mathrm{~nm}(n>500)$ (Fig. S-1). The UV-Vis spectra corroborated the TEM results and showed a small hump at around $500 \mathrm{~nm}$ suggesting the presence of very tiny size nanoparticles (Rance et al. 2008) (Fig. S-1).
Scheme 1 A schematic illustration of glucose-capped silver nanoparticles synthesis by means of heteroepitaxial growth approach and their application for optical detection of $\mathrm{Hg}^{2+}$

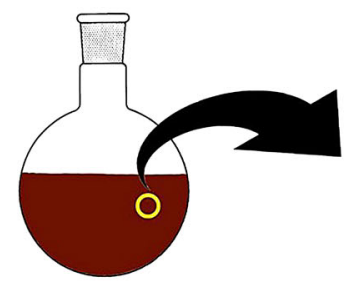

Seed AuNPs
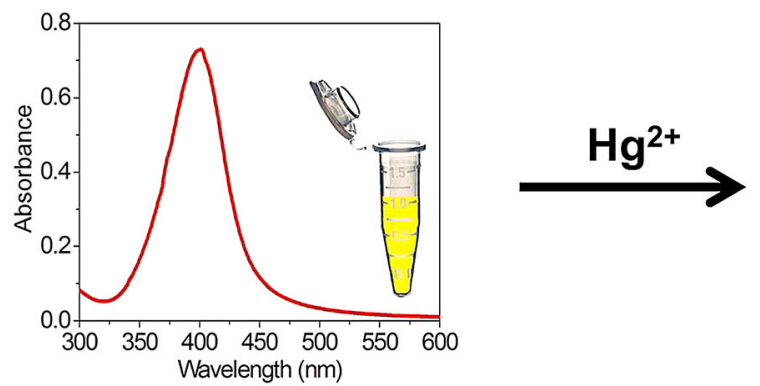

Glucose
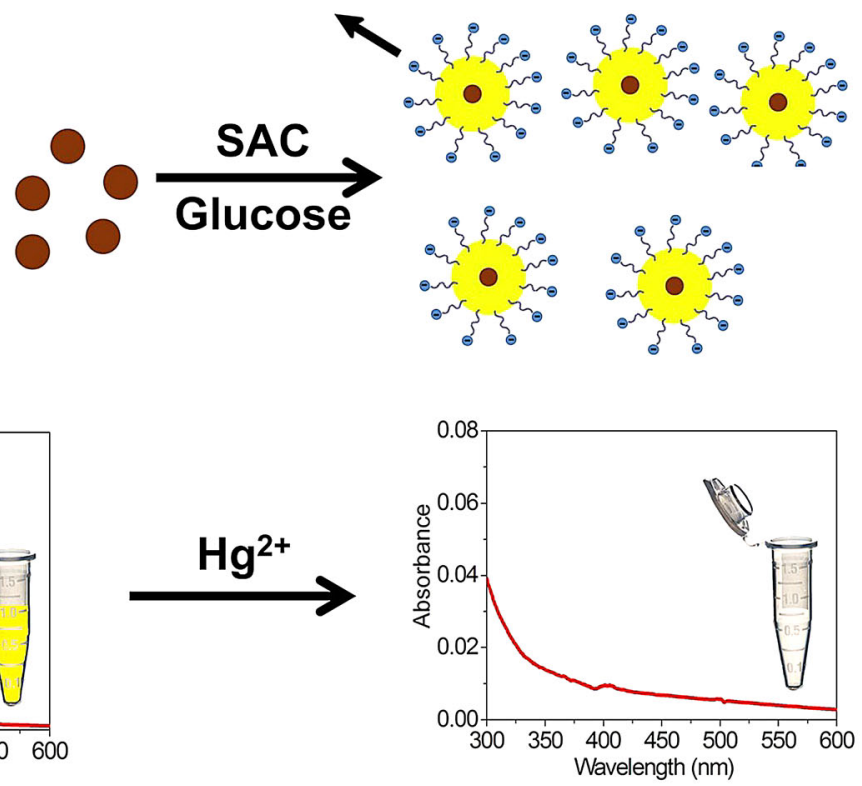
Silver nanoparticles were prepared by modified Tollens' reaction, i.e., by reducing the silver ammonia complex $\left(\left[\mathrm{Ag}\left(\mathrm{NH}_{3}\right)_{2}\right]^{+}\right)$using glucose (i.e., an aldose sugar) as a reducing agent. First, silver ammonia complex (SAC) was prepared by adding $65 \mu \mathrm{L}$ of $\mathrm{NaOH}(1 \mathrm{M})$ solution to $15 \mathrm{~mL}$ of freshly prepared aqueous silver nitrate solution $(1 \mathrm{mM})$, which instantaneously yielded a gray color precipitate. To this mixture, $80 \mu \mathrm{L}$ of freshly prepared aqueous ammonia solution $(6.25 \% \mathrm{v} / \mathrm{v})$ was added, which immediately dissolved the precipitate to give a clear solution of SAC. Thereafter, $5 \mu \mathrm{L}$ of seed AuNP solution was added to $0.3 \mathrm{~mL}$ of $\mathrm{SAC}$ solution, followed by addition of DI water to make up the final volume to $2.0 \mathrm{~mL}$. To this stirred mixture, $80 \mu \mathrm{L}$ of glucose solution (concentration $=36 \mathrm{mg} / \mathrm{mL}$ ) was added, which resulted in the change in the color of the solution from colorless to yellow color within few seconds indicating the formation of GluAgNPs.

The nanoparticles were characterized using UV-Vis spectrophotometer (Jasco Inc., Japan) and TEM (FEITecnai G220, USA). TEM grid was prepared by drop casting $10 \mu \mathrm{L}$ of colloidal solution of Glu-AgNPs onto the carbon-coated copper grid and dried using hot air oven. The size and shape distribution analysis of Glu-AgNPs was carried out using ImageJ software. The Fourier transform infrared spectroscopy (FTIR) of the Glu-AgNPs was carried out to identify the capping agent using purified nanoparticles. Prior to FTIR analysis, Glu-AgNP solution was washed by following three cycles of centrifugation (speed $4300 \mathrm{~g}$, time $20 \mathrm{~min}$ ) and re-dispersion in DI water. The zeta potential of the Glu-AgNPs was determined by means of zeta potential analyzer (90 Plus Particle Size Analyzer, Brookhaven Instruments Corporation, using ZetaPlus software).

\section{Sensing of $\mathbf{H g}^{2+}$}

For optical detection of $\mathrm{Hg}^{2+}$, aliquots of aqueous solutions containing different concentrations of $\mathrm{Hg}^{2+}$ (in the range of $100-10 \mathrm{mM}$ ) were prepared using a $10 \mathrm{mM}$ stock solution of $\mathrm{HgCl}_{2}$. A working solution of Glu-AgNPs was prepared by diluting $2 \mathrm{~mL}$ of as-prepared colloidal nanoparticle solution with $18 \mathrm{~mL}$ of DI water. Thereafter, $500 \mu \mathrm{L}$ of $\mathrm{Hg}^{2+}$ solution (of each concentration) was added to $500 \mu \mathrm{L}$ of working colloidal solution of Glu-AgNPs in separate vials and mixed thoroughly for $15 \mathrm{~min}$. Two different control samples were also prepared by mixing $500 \mu \mathrm{L}$ of DI water to equal volume of (1) working colloidal solution of Glu-AgNPs and (2) $\mathrm{HgCl}_{2}(10 \mathrm{mM})$. Thereafter, all the samples were observed for visible color change while the spectral shift and change in absorbance intensity at peak absorption wavelength were measured using UV-Visible spectrophotometer.

\section{Selectivity for $\mathrm{Hg}^{2+}$ sensing}

Since in the real environmental aqueous samples, the presence of other metal ions can interfere with the mercury detection, a non-specific interaction study was carried out using salt solution of several heavy metal ions, such as $\mathrm{Ca}^{2+}, \mathrm{Fe}^{3+}, \mathrm{Ba}^{2+}, \mathrm{Mn}^{2+}, \mathrm{Mg}^{2+}, \mathrm{Cu}^{2+}, \mathrm{Co}^{2+}, \mathrm{Cr}^{3+}, \mathrm{Ni}^{2+}$, $\mathrm{Zn}^{2+}$, and $\mathrm{Pb}^{2+}$. A $500 \mu \mathrm{L}$ of metal salt solution $(1 \mu \mathrm{M})$ was mixed with equal volume of working solution of GluAgNPs and mixed thoroughly. After $15 \mathrm{~min}$, all the samples were characterized for spectral shift and absorbance change using UV-Visible spectrophotometer.

\section{Paper-based detection of $\mathrm{Hg}^{2+}$}

To develop a paper-based colorimetric sensing strip for $\mathrm{Hg}^{2+}$ detection, $1 \mu \mathrm{L}$ of as-prepared colloidal solution of Glu-AgNPs was drop-casted on 12 different spots (spot area $=12.5 \mathrm{~mm}^{2}$ ) on a piece of Whatman filter paper no. 1 (pore size $=11 \mu \mathrm{m}$ ) followed by room temperature drying for 15 min. These Glu-AgNP-immobilized paper strips were used for mercury detection. For this, mercury solution of varying concentrations $(1,10,100,500,1,10 \mathrm{mM})$ was dispensed shot-wise (each shot volume $=1 \mu \mathrm{L}$ ), at the interval of $1 \mathrm{~min}$, on six different Glu-AgNP spots in the test line ( $\mathrm{T}$ line) until their decoloration was observed. The volume of mercury solution consumed for all the concentrations (i.e., the number of shots $\times 1 \mu \mathrm{L}$ ) for decoloration of Glu-AgNP spot was noted. For control experiments, matching volume of DI water, i.e., equivalent of volume of mercury solution consumed for decoloration of spot, was added to Glu-AgNP spot in a shot-wise fashion (shot volume $=1 \mu \mathrm{L})$ in the control line (C line).

\section{Results and discussion}

Silver nanoparticles were prepared by heteroepitaxial seedmediated growth approach using modified Tollens' reaction in which the glucose served both as reductant and stabilizer. In the presence of gold seeds, the addition of glucose resulted in yellow-colored solution within $5 \mathrm{~s}$ suggesting the formation of Glu-AgNPs. The faster synthesis of nanoparticles was attributed to the presence of pre-formed seed AuNPs, which facilitated in bypassing the burst nucleation phase and resulting in their rapid seedmediated growth. The extinction maxima of Glu-AgNPs was observed at $402 \pm 3.75 \mathrm{~nm}(n>10)$ with full width at half maximum (FWHM) value of $58.45 \pm 1.15(n>10)$ indicating the formation of monodisperse nanoparticles with very narrow size distribution (Fig. 1a). TEM image analysis corroborated the UV-Vis spectral analysis and revealed the formation of uniform, spherical-shaped 

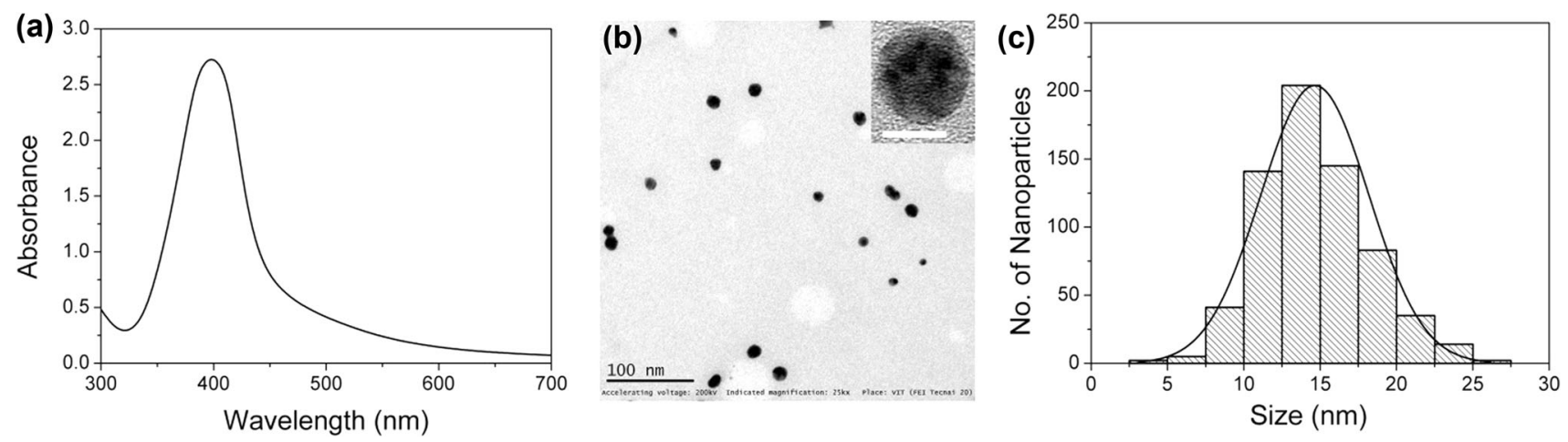

Fig. 1 a Extinction spectra, b TEM image, and c histogram of glucose-capped silver nanoparticles. Bar in inset image (b) is equal to $10 \mathrm{~nm}$

nanoparticle formation (Fig. 1b). Histogram analysis showed that the average size of Glu-AgNPs was $14.65 \pm 3.53 \mathrm{~nm} \quad(n>600)$ with more than $70 \%$ nanoparticles in the size range of $12-18 \mathrm{~nm}$ (Fig. 1c).

Since the glucose serves as both reducing agent and stabilizer, its presence as capping material was confirmed using FTIR as shown in Fig. S-2. The FTIR spectra of silver nanoparticles clearly showed the characteristic broad and strong band at $3500-3000 \mathrm{~cm}^{-1}$ due to $-\mathrm{OH}$ stretching vibrations, $-\mathrm{C}=\mathrm{O}$ stretching vibration at $1735 \mathrm{~cm}^{-1}$, and bending vibrations due to deformation of $\mathrm{OCH}$ and $\mathrm{COH}$ at 1367 and $1215 \mathrm{~cm}^{-1}$, respectively (Ibrahim et al. 2006). Further, negative zeta potential value (i.e., $-23.7 \pm 5.1 \mathrm{mV}$ ) also indicated the presence of glucose as capping agent.

\section{Optical detection of $\mathbf{H g}^{2+}$}

The interaction of Glu-AgNPs with $\mathrm{Hg}^{2+}$ resulted in a prominent visible color change, from yellow to colorless, particularly at high concentration of $\mathrm{Hg}^{2+}$ (i.e., $\geq 10 \mu \mathrm{M}$ ). At further lower concentrations, the color change could not be noticed easily through the naked eye, but optical change could be measured by UV-visible spectrophotometer. A decrease in absorbance intensity along with a blue shift in the peak absorption wavelength was also noticed with an increase in $\mathrm{Hg}^{2+}$ ion concentration in the range of 100 to
$10 \mathrm{mM}$ (Fig. 2a). At high concentration, the disappearance of characteristic absorption peak of silver nanoparticles was observed resulting in a clear solution with zero or nearly zero absorbance intensity. The sensor response, i.e., percent absorbance ratio $\left(A \times 100 / A_{0}\right)$, where $A_{0}$ is the absorbance of control sample having only Glu-AgNPs and $A$ is the absorbance of Glu-AgNP solution after interaction with $\mathrm{Hg}^{2+}$ sample, was observed to be linear $\left(R^{2}=0.99\right)$ (Fig. 2b).

\section{Mechanism of $\mathrm{Hg}^{2+}$ sensing}

Upon interaction of Glu-AgNPs with $\mathrm{Hg}^{2+}$ solution, the blue shift and decrease in absorbance intensity can possibly be attributed to (1) the facilitation of $\mathrm{Hg}^{2+}$ complexation with Glu-AgNPs via electrostatic interaction between cationic mercury ions and anionic glucose capping material (Kennedy et al. 2014; Vaseem et al. 2013) and (2) redox etching of Glu-AgNPs by means of $\mathrm{Hg}^{2+}$ resulting in $\mathrm{Ag}-$ $\mathrm{Hg}$ amalgam formation. This is due to the fact that standard electrode potential of $\mathrm{Hg}^{2+} / \mathrm{Hg}(0.85 \mathrm{~V})$ is higher than the $\mathrm{Ag}^{+} / \mathrm{Ag}(0.80 \mathrm{~V})$, which causes redox etching of silver nanoparticles. This was evidenced by the TEM image analysis of $\mathrm{Hg}^{2+}$ (concentration $=1 \mu \mathrm{M}$ )-treated GluAgNPs (Fig. 3a) where few silver nanoparticles of smaller size could be observed. In addition, the hollow cavity could be discerned easily in more than $70 \%$ of the mercury-
Fig. 2 a UV-Visible spectra of colloidal Glu-AgNP solution before and after interaction with different concentrations of mercury solution and $\mathbf{b}$ semi-log linear correlation graph of different concentrations of $\mathrm{Hg}^{2+}$ vs. percent absorbance ratio
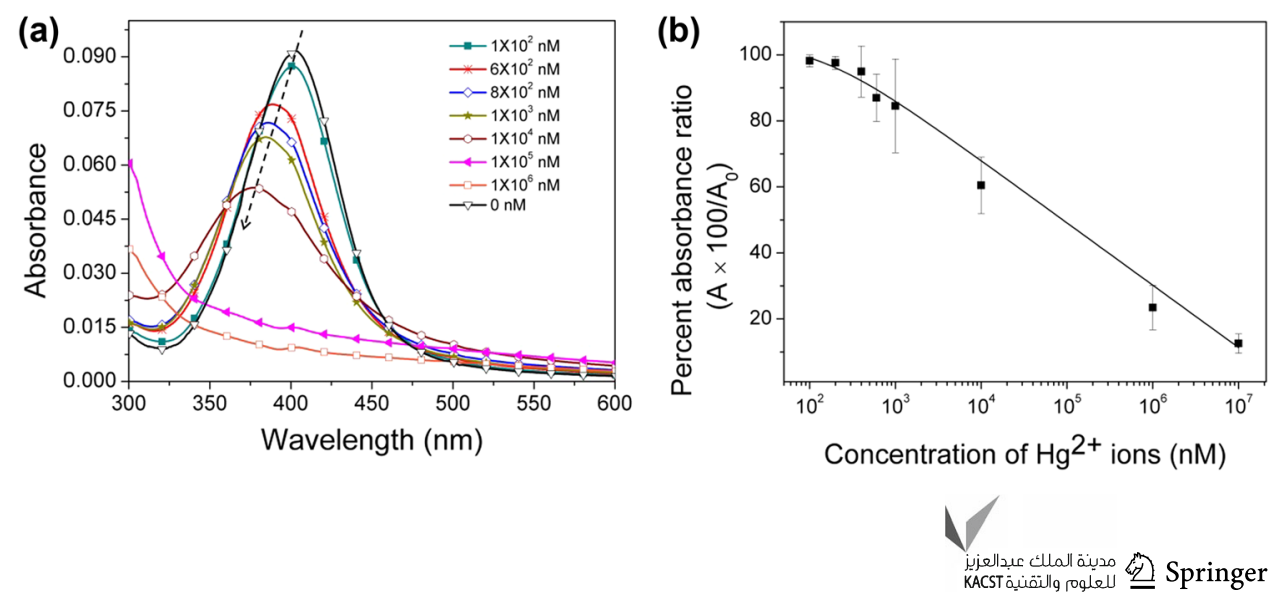


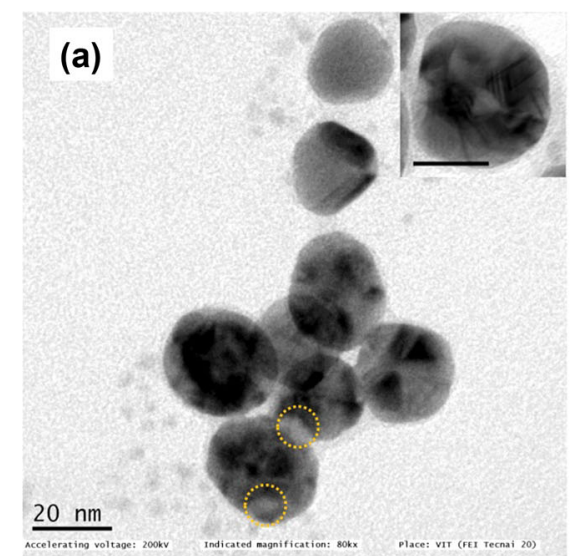

Fig. 3 a TEM image of silver nanoparticles after treatment with mercury solution (concentration $=1 \mu \mathrm{M}$ ) showing the presence of pores in the nanoparticles and deformation of shape probably due to etching of nanoparticles and deposition of mercury, Inset image shows a noticeable presence of etched pore in the center of a

treated nanoparticles ( $n>150$ nanoparticles) further suggesting the uniform etching of silver nanoparticles. A slight increase in the size along with shape deformation and hollow cavities were also noticed in few nanoparticles. This could be due to the deposition of mercury $\left(\mathrm{Hg}^{0}\right)$ on the etched or partially etched silver nanoparticles by means of amalgam formation. This was further evidenced by energydispersive X-ray analysis (EDX) of $\mathrm{Hg}^{2+}$-treated GluAgNPs which confirmed the presence of mercury in the nanoparticle sample (Fig. 3b). In addition, aggregates of silver nanoparticles were also observed (image not shown), which were probably formed due to rapid amalgam formation either locally (i.e., at low $\mathrm{Hg}^{2+}$ concentration causing negligible visible change in color) or globally (i.e., at high concentration causing immediate aggregation of all the nanoparticles causing decoloration).

To study the reaction kinetics between Glu-AgNPs and $\mathrm{Hg}^{2+}$, a time-dependent absorbance change (at $\lambda_{\max }=402 \mathrm{~nm}$ ) of the reaction mixture was monitored. The reaction kinetic study was performed for four different concentrations of mercury ions, i.e., 200, 600, 1, $10 \mathrm{mM}$, while the nanoparticle concentration was kept constant in all the batches. A $500 \mu \mathrm{L}$ of working solution of GluAgNPs was mixed with equal volume of mercury ions in the quartz cuvette, and was immediately used for studying the absorbance measurement at $402 \mathrm{~nm}$. The spectral response measurement was started after $2 \mathrm{~s}$ of the mixing of nanoparticles and $\mathrm{Hg}^{2+}$ ions, as it was required for loading the cuvette in the spectrophotometer and software operation. The course of reaction was monitored till the completion of reaction, i.e., saturation in absorbance response, and the data were recorded at intervals of $0.05 \mathrm{~s}$. The procedure was repeated for all four concentrations of

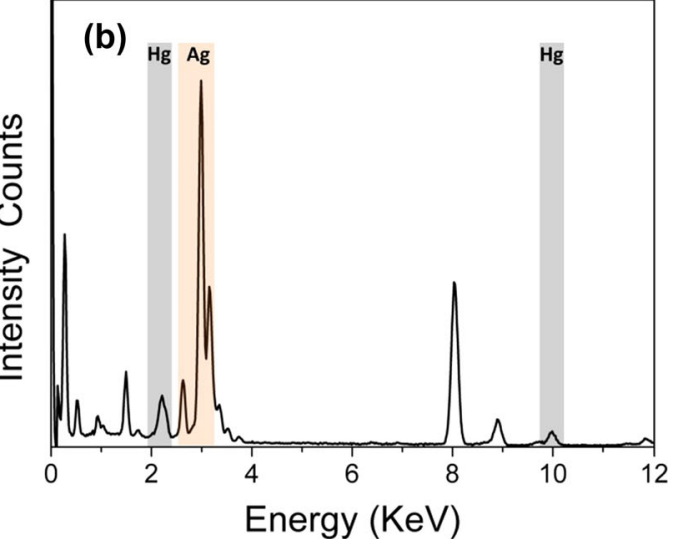

nanoparticle after treatment with mercury sample. The scale bar represents $20 \mathrm{~nm}$ in the inset image; b energy-dispersive X-ray spectrum of silver nanoparticles treated with $\mathrm{Hg}^{2+}$ depicting the presence of mercury which is probably due to the formation of $\mathrm{Ag}$ $\mathrm{Hg}$ amalgam

mercury ions and one control sample, where only DI water was added to working solution of Glu-AgNPs.

Figure 4 depicts the reaction kinetics curve between Glu-AgNPs and $\mathrm{Hg}^{2+}$ ions of varying concentrations. It is evident that at very high concentration of $\mathrm{Hg}^{2+}$ (i.e., $10 \mathrm{mM}$ and $1 \mu \mathrm{M})$, the reaction time was very fast resulting in a significant drop in the absorbance intensity from the initial time point. In contrast, at lower concentration of $\mathrm{Hg}^{2+}$ (i.e., 200 and $600 \mathrm{nM}$ ), a time-dependent decrease in the absorbance intensity was observed, which reached saturation in 60-80 s. At low $\mathrm{Hg}^{2+}$ concentration, the reaction kinetics followed the first-order behavior. This suggests that the rate of Glu-AgNP etching reaction is dependent on the concentration of mercury ions and it is

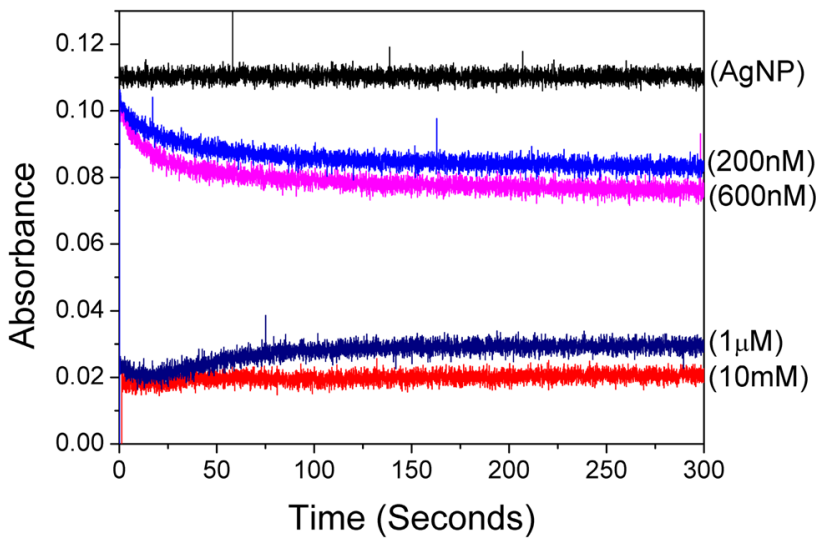

Fig. 4 A concentration-dependent reaction kinetic curve displaying the first-order reaction between Glu-AgNPs and $\mathrm{Hg}^{2+}$ at 200 and $600 \mathrm{nM}$ concentrations. At 1 and $10 \mathrm{mM}$, the reaction rate was very high, as a consequence, significantly low absorption value was observed at zero time point. The control sample (Glu-AgNPs) did not show any change in absorbance during the course of interaction 
very fast at high concentration of mercury (i.e., $\geq 1 \mu \mathrm{M})$. In contrast, at low concentration, i.e., $\leq 600 \mathrm{nM}$, it is relatively slow and the analysis takes at least $60 \mathrm{~s}$. This analysis time achieved in our study was relatively very short compared to the previously reported studies where the time taken was almost $1 \mathrm{~h}$ (Farhadi et al. 2012; Jeevika and Shankaran 2016; Lin et al. 2010). The high reaction rate was attributed to the presence of glucose capping, which is anionic in nature and facilitated the electrostatic attraction and etching of cationic mercury ions in the aqueous medium. This was further investigated by comparing the mercury-sensing performance of Glu-AgNPs with citratecapped silver nanoparticles (Cit-AgNPs). The Cit-AgNPss were prepared as reported by Agnihotri et al. (2014) and used to detect mercury similar to the Glu-AgNP. At concentration of 1 and $100 \mu \mathrm{M}$, of mercury, the sensor response, i.e., percent absorbance ratio, was found to be two- to threefold enhanced for Glu-AgNPs compared to the Cit-AgNPs. This suggested that the presence of glucose capping facilitates the complexation of $\mathrm{Hg}^{2+}$ solution (Huljev et al. 1983) and thus improves the sensitivity.

The unmodified Glu-AgNPs as a probe could detect $100 \mathrm{nM}$ (eq. to $20 \mathrm{ppb}$ ) of $\mathrm{Hg}^{2+}$ in aqueous sample. The sensitivity of the probes was found to be comparable (Jeevika and Shankaran 2016) or one order better (Ma et al. 2016; Rithesh Raj et al. 2016) compared to the recently developed various nanoanalytical methods. Although the sensitivity was observed to be relatively less compared to the electrochemical sensor, this relies on sensor matrices consisting of sensitive and costly DNA/imprinted polymer/ other complex nanohybrid structures (Zhou et al. 2016;
Bahrami et al. 2015), which limit their field deployability. In addition, the present approach offers the advantages of shorter assay time, low cost, user-friendliness as it does not require any pre-treatment or additional reagents during the assay.

To estimate the selectivity, the assay was performed with various metal salt solutions, such as $\mathrm{Ca}^{2+}, \mathrm{Fe}^{3+}, \mathrm{Ba}^{2+}$, $\mathrm{Mn}^{2+}, \mathrm{Mg}^{2+}, \mathrm{Cu}^{2+}, \mathrm{Co}^{2+}, \mathrm{Cr}^{3+}, \mathrm{Ni}^{2+}, \mathrm{Zn}^{2+}$, and $\mathrm{Pb}^{2+}$. No significant change in color, extinction maxima, and absorbance intensity was observed at $100 \mu \mathrm{M}$ concentrations of these ions indicating a very high selectivity of the Glu-AgNP probes towards mercury sensing by virtue of specific redox etching of nanoparticles and $\mathrm{Ag}-\mathrm{Hg}$ amalgam formation (Fig. S-3).

\section{Paper-based detection of $\mathbf{H g}^{2+}$}

To ensure the field deployability of the Glu-AgNP probebased $\mathrm{Hg}^{2+}$ sensing, we attempted to develop a paperbased sensing strip. Figure 5 depicts the photographic image captured just after the disappearance of yellow spots of Glu-AgNPs (in $\mathrm{T}$ line) with their respective control sample (in $\mathrm{C}$ line). At $10 \mathrm{mM} \mathrm{Hg}^{2+}$ concentration, just after addition of first shot, Glu-AgNP spot became colorless, while the control sample did not show any color change (Fig. 5b). For other concentrations, i.e., $1 \mathrm{mM}, 500$, 100,10 , and $1 \mu \mathrm{M}$, the numbers of shots consumed for decoloration were 2, 3, 30, 60, and 120, respectively. For all the control samples, no visible change in yellow color of Glu-AgNP spots was observed. It is evident that as the concentration of mercury was decreased, the required

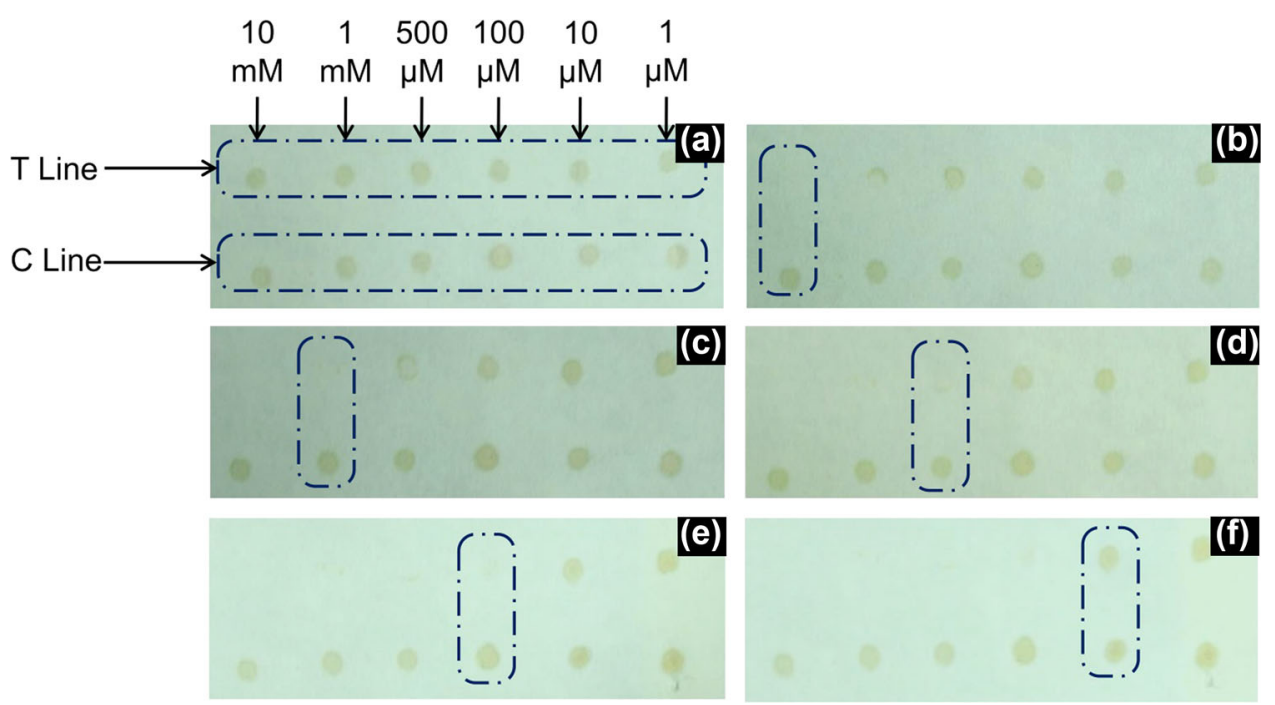

Fig. 5 Photographic image of paper-based mercury-sensing strip developed by drop casting of glucose-capped silver nanoparticles in circular spots; a sensing strip showing the test $(T)$ line, where mercury sample of different concentrations were drop-wise added, and the control $(C)$ line, where DI water was added and used as a control.
Photographic images showing decoloration of yellow spots in $T$ line after interaction with b $1 \mu \mathrm{L}$ of $10 \mathrm{mM}$, c $2 \mu \mathrm{L}$ of $1 \mathrm{mM}$, d $3 \mu \mathrm{L}$ of $500 \mu \mathrm{M}$, e $30 \mu \mathrm{L}$ of $100 \mu \mathrm{M}$, and f $60 \mu \mathrm{L}$ of $10 \mu \mathrm{M}$ concentration of mercury sample. The mercury solution was added in a shot-wise manner at the interval of $1 \mathrm{~min}$, where volume of each shot was $1 \mu \mathrm{L}$ 
number of shots (or the sample volume) and the analysis time increased. This was due to the decreased availability of mercury ions, which slowed down the reaction rate. Under the sub-optimal experimental conditions, the present paper-based sensor strip could detect $1 \mu \mathrm{M}$ of mercury ion within $2 \mathrm{~h}$. At further lower concentration $(<1 \mu \mathrm{M})$, the naked eye detection can be achieved, but it would require relatively more time as the sample is added drop-wise followed by air drying. This problem can be solved by applying a pre-concentrator mechanism which will increase the concentration of mercury ions either by means of adsorption or chelation and reduce the reaction time for a visual detectable change (Han et al. 2016). However, incorporation of these tools or strategies will increase the complexity of sensor and cost of assay/device.

\section{Conclusion}

In this study we have investigated the potential of glucose-capped silver nanoparticles as smart probes for sensing of $\mathrm{Hg}^{2+}$ in aqueous sample. The silver nanoparticles were prepared by heteroepitaxial seedmediated growth method using tiny gold nanoparticles as seed. The silver was grown on these seed particles using a modified Tollens' reaction. In this process glucose was used as both reducing agent and stabilizer which was confirmed using FTIR and zeta potential measurement. The resultant Glu-AgNPs were observed to be spherical shaped with very narrow size and shape distribution. Upon interaction of as-prepared Glu-AgNPs with $\mathrm{Hg}^{2+}$, a prominent color change with spectral shift and change in peak absorption intensity were observed as a function of mercury concentration. The reaction rate was found to be dependent on mercury concentration, i.e., higher the concentration of $\mathrm{Hg}^{2+}$, faster is the reaction rate and thus shorter is the analysis time. The limit of detection was found to be $100 \mathrm{nM}$ with good linearity in the concentration range of $100 \mathrm{nM}-10 \mathrm{mM}$. Further, we demonstrated the potential of Glu-AgNP-based paper sensing strip which could detect $1 \mu \mathrm{M}$ of $\mathrm{Hg}^{2+}$ under sub-optimal conditions. The Glu-AgNP probes offer the advantages of simple sensing mechanism, rapid response, cost effectiveness, free from the use of any linker, chemical or biochemical and polymer, etc., high specificity, naked eye detection (in paper-based assay) and environment friendliness. The current strategy can be deployed at field condition due to it robustness, high selectivity and eco-friendly disposal. In addition, the sensitivity can be further improved by employing a preconcentrator mechanism to increase the $\mathrm{Hg}^{2+}$ concentration before interaction with silver nanoparticles.
Acknowledgements The authors would like to thank Science and Engineering and Research Board (project code YSS/2014/000003), Department of Science and Technology, Government of India for the funding.

Open Access This article is distributed under the terms of the Creative Commons Attribution 4.0 International License (http:// creativecommons.org/licenses/by/4.0/), which permits unrestricted use, distribution, and reproduction in any medium, provided you give appropriate credit to the original author(s) and the source, provide a link to the Creative Commons license, and indicate if changes were made.

\section{References}

Agnihotri S, Mukherji S, Mukherji S (2014) Size-controlled silver nanoparticles synthesized over the range 5-100 $\mathrm{nm}$ using the same protocol and their antibacterial efficacy. RSC Adv 4(8):3974-3983. doi:10.1039/c3ra44507k

Alex SA, Chandrasekaran N, Mukherjee A (2016) State-of-the-art strategies for the colorimetric detection of heavy metals using gold nanorods based on aspect ratio reduction. Anal Methods 8(10):2131-2137. doi:10.1039/c5ay03428k

Bahrami A, Besharati-Seidani A, Abbaspour A, Shamsipur M (2015) A highly selective voltammetric sensor for nanomolar detection of mercury ions using a carbon ionic liquid paste electrode impregnated with novel ion imprinted polymeric nanobeads. Mater Sci Eng C 48:205-212. doi:10.1016/j.msec.2014.12.005

Barin JS, Tischer B, Picoloto RS, Antes FG, da Silva FEB, Paula FR et al (2014) Determination of toxic elements in tricyclic active pharmaceutical ingredients by ICP-MS: a critical study of digestion methods. J Anal At Spectrom 29(2):352-358. doi:10. 1039/c3ja50334h

Bhattacharjee Y, Chakraborty A (2014) Label-free cysteaminecapped silver nanoparticle-based colorimetric assay for $\mathrm{Hg}$ (II) detection in water with subnanomolar exactitude. ACS Sustain Chem Eng 2(9):2149-2154. doi:10.1021/sc500339n

Botasini S, Heijo G, Méndez E (2013) Toward decentralized analysis of mercury (II) in real samples. A critical review on nanotechnology-based methodologies. Anal Chim Acta 800:1-11. doi:10. 1016/j.aca.2013.07.067

Clarkson TW, Magos L (2006) The toxicology of mercury and its chemical compounds. Crit Rev Toxicol 36(8):609-662. doi:10. 1080/10408440600845619

Cui L, Wu J, Ju H (2015) Electrochemical sensing of heavy metal ions with inorganic, organic and bio-materials. Biosens Bioelectron 63:276-286. doi:10.1016/j.bios.2014.07.052

Ding N, Zhao H, Peng W, He Y, Zhou Y, Yuan L et al (2012) A simple colorimetric sensor based on anti-aggregation of gold nanoparticles for $\mathrm{Hg}^{2+}$ detection. Coll Surf A 395:161-167. doi:10.1016/j.colsurfa.2011.12.024

Ding Y, Wang S, Li J, Chen L (2016) Nanomaterial-based optical sensors for mercury ions. TrAC Trends Anal Chem 82:175-190. doi: $10.1016 / \mathrm{j}$. trac. 2016.05 .015

Duan J, Yin H, Wei R, Wang W (2014) Facile colorimetric detection of $\mathrm{Hg}^{2+}$ based on anti-aggregation of silver nanoparticles. Biosens Bioelectron 57:139-142. doi:10.1016/j.bios.2014.02. 007

Duff DG, Baiker A, Edwards PP (1993) A new hydrosol of gold clusters. 1. Formation and particle size variation. Langmuir 9(9):2301-2309. doi:10.1021/la00033a010 
Fan Y, Liu Z, Wang LE, Zhan J (2009) Synthesis of starch-stabilized ag nanoparticles and $\mathrm{Hg}^{2+}$ recognition in aqueous media. Nanoscale Res Lett 4(10):1230. doi:10.1007/s11671-009-9387-6

Farhadi K, Forough M, Molaei R, Hajizadeh S, Rafipour A (2012) Highly selective $\mathrm{Hg}^{2+}$ colorimetric sensor using green synthesized and unmodified silver nanoparticles. Sens Actuators B Chem 161(1):880-885. doi:10.1016/j.snb.2011.11.052

Govindhan M, Adhikari B-R, Chen A (2014) Nanomaterials-based electrochemical detection of chemical contaminants. RSC Adv 4(109):63741-63760. doi:10.1039/c4ra10399h

Graeme KA, Pollack MDFCV (1998) Heavy metal toxicity, part I: arsenic and mercury. J Emerg Med 16(1):45-56. doi:10.1016/ S0736-4679(97)00241-2

Guo L, Jackman JA, Yang H-H, Chen P, Cho N-J, Kim D-H (2015) Strategies for enhancing the sensitivity of plasmonic nanosensors. Nano Today 10(2):213-239. doi:10.1016/j.nantod.2015.02. 007

Han FX, Patterson WD, Xia Y, Sridhar BBM, Su Y (2006) Rapid determination of mercury in plant and soil samples using inductively coupled plasma atomic emission spectroscopy, a comparative study. Water Air Soil Pollut 170(1):161-171. doi:10.1007/s11270-006-3003-5

Han SI, Hwang KS, Kwak R, Lee JH (2016) Microfluidic paper-based biomolecule preconcentrator based on ion concentration polarization. Lab Chip 16(12):2219-2227. doi:10.1039/c6lc00499g

Henglein A, Brancewicz C (1997) Absorption spectra and reactions of colloidal bimetallic nanoparticles containing mercury. Chem Mater 9(10):2164-2167. doi:10.1021/cm970258x

Huljev D, Džajo M, Kristić N, Strohal P (1983) The interaction of mercury(II) ions with sugars and amino acids. Int J Environ Anal Chem 15(1):53-59. doi:10.1080/03067318308071913

Ibrahim M, Alaam M, El-Haes H, Jalbout AF, Leon AD (2006) Analysis of the structure and vibrational spectra of glucose and fructose. Eclét Quím 31:15-21

Jeevika A, Shankaran DR (2016) Functionalized silver nanoparticles probe for visual colorimetric sensing of mercury. Mater Res Bull 83:48-55. doi:10.1016/j.materresbull.2016.05.029

Karthiga D, Rajeshwari A, Chakravarty S, Chandrasekaran N, Mukherjee A (2016) Determination of mercury(II) ions in aqueous solution using silver nanorods as a probe. Anal Methods 8(18):3756-3762. doi:10.1039/c6ay00882h

Kennedy DC, Orts-Gil G, Lai C-H, Müller L, Haase A, Luch A et al (2014) Carbohydrate functionalization of silver nanoparticles modulates cytotoxicity and cellular uptake. J Nanobiotechnol 12(1):59. doi:10.1186/s12951-014-0059-Z

Lin C-Y, Yu C-J, Lin Y-H, Tseng W-L (2010) Colorimetric sensing of silver(I) and mercury(II) ions based on an assembly of tween 20-stabilized gold nanoparticles. Anal Chem 82(16):6830-6837. doi:10.1021/ac1007909

Liu H, Yang L, Ma H, Qi Z, Liu J (2011) Molecular sensitivity of DNA-Ag-PATP hybrid on optical activity for ultratrace mercury analysis. Chem Commun 47(33):9360-9362. doi:10.1039/ c1cc13336e

Ma Y, Pang Y, Liu F, Xu H, Shen X (2016) Microwave-assisted ultrafast synthesis of silver nanoparticles for detection of $\mathrm{Hg}^{2+}$. Spectrochim Acta Part A 153:206-211. doi:10.1016/j.saa.2015. 08.004

Mao S, Chang J, Zhou G, Chen J (2015) Nanomaterial-enabled rapid detection of water contaminants. Small 11(40):5336-5359. doi:10.1002/smll.201500831

Martinsson E, Otte MA, Shahjamali MM, Sepulveda B, Aili D (2014) Substrate effect on the refractive index sensitivity of silver nanoparticles. J Phys Chem C 118(42):24680-24687. doi:10. 1021/jp5084086

Puk R, Weber JH (1994) Critical review of analytical methods for determination of inorganic mercury and methylmercury compounds. Appl Organomet Chem 8(4):293-302. doi:10. 1002/aoc.590080404

Rajeshwari A, Karthiga D, Chandrasekaran N, Mukherjee A (2016) Anti-aggregation-based spectrometric detection of $\mathrm{Hg}(\mathrm{II})$ at physiological $\mathrm{pH}$ using gold nanorods. Mater Sci Eng C 67:711-716. doi:10.1016/j.msec.2016.05.066

Ramesh GV, Radhakrishnan TP (2011) A Universal sensor for mercury (Hg, HgI, HgII) based on silver nanoparticle-embedded polymer thin film. ACS Appl Mater Interfaces 3(4):988-994. doi:10.1021/am200023w

Rance GA, Marsh DH, Khlobystov AN (2008) Extinction coefficient analysis of small alkanethiolate-stabilised gold nanoparticles. Chem Phys Lett 460(1-3):230-236. doi:10.1016/j.cplett.2008. 06.010

Ren W, Zhu C, Wang E (2012) Enhanced sensitivity of a direct SERS technique for $\mathrm{Hg}^{2+}$ detection based on the investigation of the interaction between silver nanoparticles and mercury ions. Nanoscale 4(19):5902-5909. doi:10.1039/c2nr31410j

Rithesh Raj D, Prasanth S, Vineeshkumar TV, Sudarsanakumar C (2016) Surface Plasmon Resonance based fiber optic sensor for mercury detection using gold nanoparticles PVA hybrid. Opt Commun 367:102-107. doi:10.1016/j.optcom.2016.01.027

Rooney JPK (2007) The role of thiols, dithiols, nutritional factors and interacting ligands in the toxicology of mercury. Toxicology 234(3):145-156. doi:10.1016/j.tox.2007.02.016

Sun Z, Du J, Jing C (2016) Recent progress in detection of mercury using surface enhanced Raman spectroscopy-A review. J Environ Sci 39:134-143. doi:10.1016/j.jes.2015.11.009

Tao Y, Lin Y, Huang Z, Ren J, Qu X (2012) Poly(acrylic acid)templated silver nanoclusters as a platform for dual fluorometric turn-on and colorimetric detection of mercury (II) ions. Talanta 88:290-294. doi:10.1016/j.talanta.2011.10.043

Tchounwou PB, Ayensu WK, Ninashvili N, Sutton D (2003) Review: environmental exposure to mercury and its toxicopathologic implications for public health. Environ Toxicol 18(3):149-175. doi:10.1002/tox.10116

Tharion J, Satija J, Mukherji S (2015) Facile synthesis of size-tunable silver nanoparticles by heteroepitaxial growth method for efficient NIR SERS. Plasmonics 10(4):753-763. doi:10.1007/ s11468-014-9862-5

Trasande L, Landrigan PJ, Schechter C (2005) Public health and economic consequences of methyl mercury toxicity to the developing brain. Environ Health Perspect 113(5):590-596. doi:10.1289/ehp.7743

Vallant B, Kadnar R, Goessler W (2007) Development of a new HPLC method for the determination of inorganic and methylmercury in biological samples with ICP-MS detection. J Anal At Spectrom 22(3):322-325. doi:10.1039/b615463h

Vaseem M, Tripathy N, Khang G, Hahn Y-B (2013) Green chemistry of glucose-capped ferromagnetic hcp-nickel nanoparticles and their reduced toxicity. RSC Adv 3(25):9698-9704. doi:10.1039/ c3ra40462e

Wang Y, Yang F, Yang X (2010) Colorimetric detection of mercury(II) ion using unmodified silver nanoparticles and mercury-specific oligonucleotides. ACS Appl Mater Interfaces 2(2):339-342. doi:10.1021/am9007243

Yuan X, Wen S, Shen M, Shi X (2013) Dendrimer-stabilized silver nanoparticles enable efficient colorimetric sensing of mercury ions in aqueous solution. Anal Methods 5(20):5486-5492. doi:10.1039/c3ay41331d

Zhou G, Chang J, Pu H, Shi K, Mao S, Sui X et al (2016) Ultrasensitive mercury ion detection using dna-functionalized molybdenum disulfide nanosheet/gold nanoparticle hybrid fieldeffect transistor device. ACS Sens 1(3):295-302. doi:10.1021/ acssensors.5b00241 